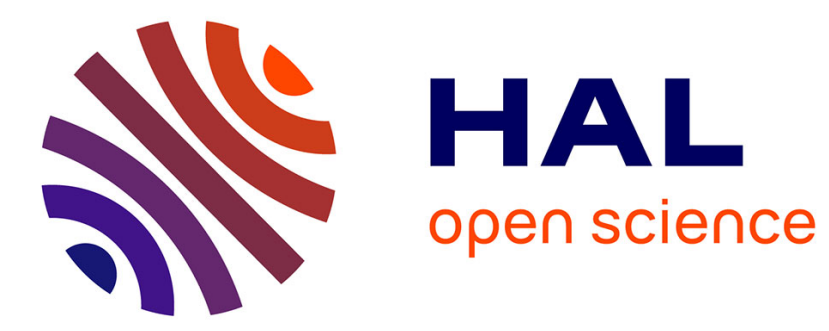

\title{
The TWINS-LAD mission: Observations of terrestrial Lyman-? fluxes
}

\author{
H. U. Nass, J. H. Zoennchen, G. Lay, H. J. Fahr
}

\section{To cite this version:}

H. U. Nass, J. H. Zoennchen, G. Lay, H. J. Fahr. The TWINS-LAD mission: Observations of terrestrial Lyman-? fluxes. Astrophysics and Space Sciences Transactions, 2006, 2 (1), pp.27-31. hal-00297477

\section{HAL Id: hal-00297477 \\ https://hal.science/hal-00297477}

Submitted on 27 Apr 2006

HAL is a multi-disciplinary open access archive for the deposit and dissemination of scientific research documents, whether they are published or not. The documents may come from teaching and research institutions in France or abroad, or from public or private research centers.
L'archive ouverte pluridisciplinaire HAL, est destinée au dépôt et à la diffusion de documents scientifiques de niveau recherche, publiés ou non, émanant des établissements d'enseignement et de recherche français ou étrangers, des laboratoires publics ou privés. 


\title{
The TWINS-LAD mission: \\ Observations of terrestrial Lyman- $\alpha$ fluxes
}

\author{
H. U. Nass, J. H. Zoennchen, G. Lay, and H. J. Fahr \\ Inst. for Astrophysics and Space Research, University of Bonn
}

Received: 6 December 2005 - Revised: 17 February 2006 - Accepted: 27 February 2006 - Published: 27 April 2006

\begin{abstract}
The TWINS project (Two Wide-angle Imaging Neutral-atom Spectrometers) is mainly devoted to measure high energy neutral atoms (ENAs) originating via charge exchange of protons with geocoronal hydrogen atoms in the plasmasphere and magnetosphere. In order to unfold the local ion density along the line-of-sight (LOS) from the integrated ENA flux measurements, a good knowledge of the geocoronal hydrogen density distribution is needed. Therefore, two Lyman- $\alpha$ detectors (LADs) - designed and calibrated by the authors - were added to the TWINS package. These detectors register line-integrated Lyman- $\alpha$ resonance emission intensities which then can be used to get the actual local hydrogen densities with the help of a numerical inversion routine.
\end{abstract}

\section{Instrumentation}

The TWINS mission consists of two spacecraft. Each one will be 3-axis stabilized and approximately nadir pointed. The orbits are so called Molniya orbits with an inclination of $63.4^{\circ}$, a perigee of roughly $1000 \mathrm{~km}$ and an apogee of 7.2 earth radii (Fig. 1) The first spacecraft will be launched at the beginning of 2006 and the second one in 2007. The expected minimal lifetime of the instruments is about 3 years, so that for at least 1.5 years both spacecraft will be measuring simultaneously. A stereo view will be accomplished by a $180^{\circ}$ shift in the longitudes of the rising nodes of the two satellite orbits. A sketch of the whole scientific instrumentation is shown in Fig. 2. Each instrument consists of two ENA-particle detectors and two Lyman- $\alpha$ detectors (LADs). The Lyman- $\alpha$ detectors are arranged in a plane parallel to the two ENA-particle sensor heads and inclined to the rotation axis of the actuator by $\pm 40^{\circ}$.

Correspondence to: H. U. Nass (unass@ astro.uni-bonn.de)

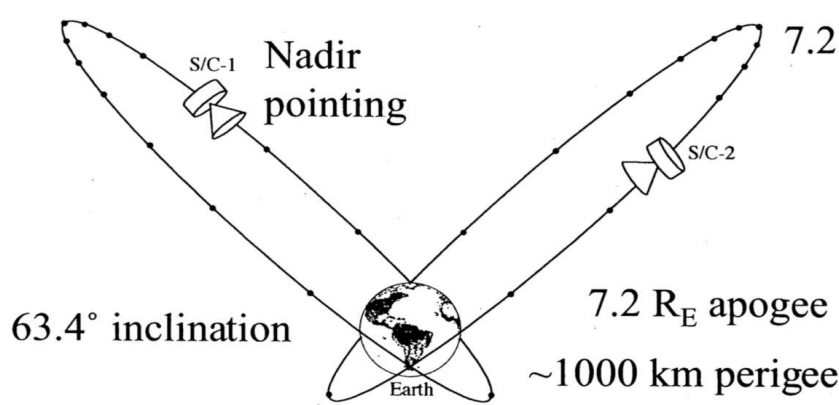

Fig. 1. Orbits of the two TWINS satellites.

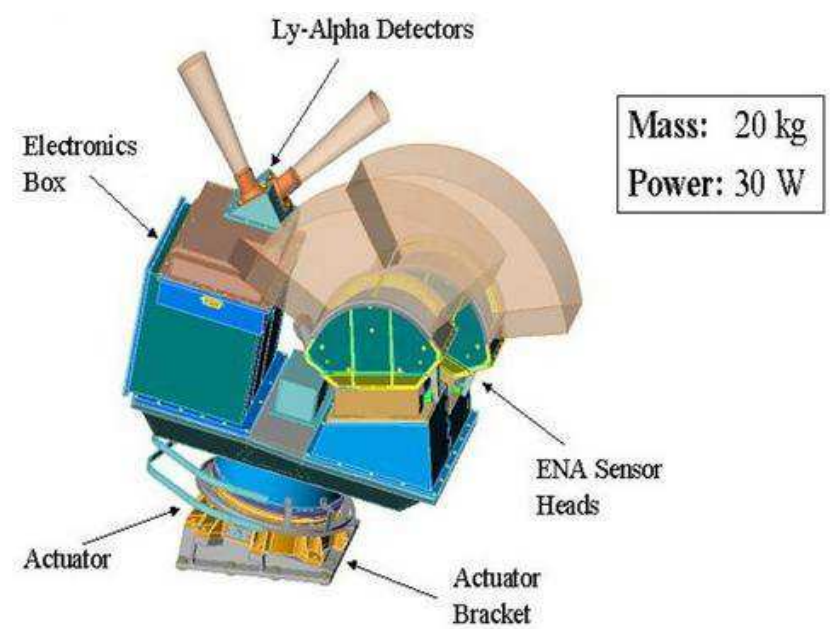

Fig. 2. Sketch of the scientific payload. The light pink areas above the detectors indicate the field of view of the respective sensor.

Hence, the Lyman- $\alpha$ detectors are pointing almost to the same directions as the TWINS particle imagers. During the mission the actuator will rotate the instrument in a wind-

Published by Copernicus GmbH on behalf of the Arbeitsgemeinschaft Extraterrestrische Forschung e.V. 


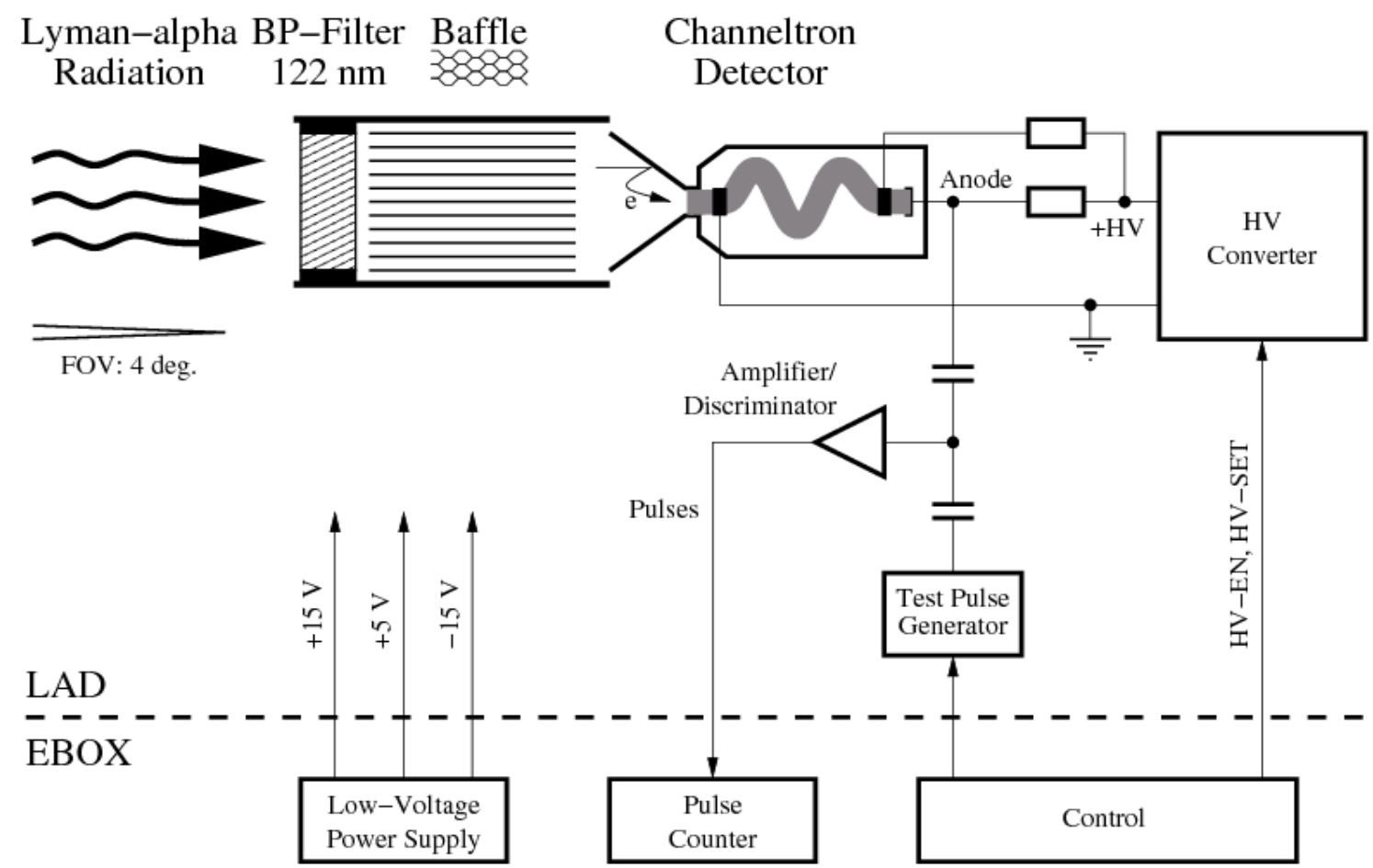

Fig. 3. Sketch of a LAD sensor with light path and electric circuitry.

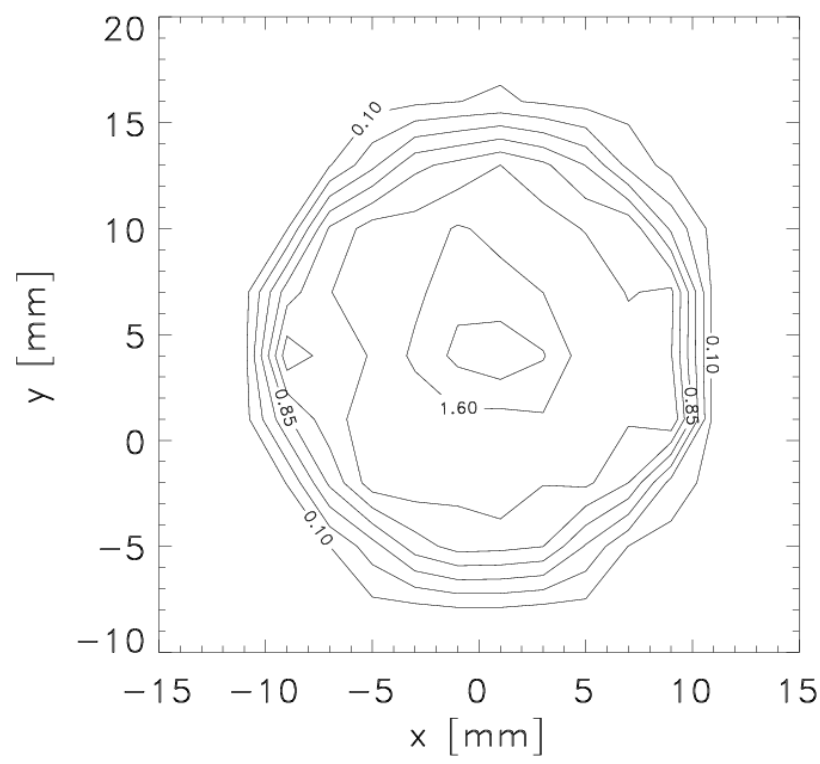

Fig. 4. Local quantum efficiency $\mathrm{QE}^{\prime}$ at Lyman- $\alpha$ measured over one LAD sensor surface (without filter). The numbers on the contours are in cts per 100 photons, i.e. percent.

shield wiper mode, i.e. back and forth, through $180^{\circ}$ with a rotation speed of approximately $3^{\circ}$ per second with the rotation axis pointing to the center of the earth. Since the two sensors are oriented symmetrically with respect to the rotation axis, a full circle is mapped. At the two turning points, i.e. at $0^{\circ}$ and $180^{\circ}$, the Lyman- $\alpha$ sensor $\# 1$ will point to the same direction as sensor \#2 in the opposite position, and vice versa, thus enabling a check of the relative calibration of the two sensors. An absolute in-flight re-calibration may also be possible, if a bright star with a known spectrum near Lyman$\alpha$ will be seen accidentally.

In order to avoid possible hazard caused by the radiation belts all scientific instruments will be switched off below an orbital radius of 4.5 earth radii. Because the $\pm 40^{\circ}$ inclination of the field of view of the LAD sensors with respect to the rotation axis the closest approach of the LOS to the earth is approximately 2.9 earth radii. It is not yet decided whether or not the instruments will be switched on at lower heights again towards the end of the mission.

Figure 3 shows the measurement principle of each sensor: The Lyman- $\alpha$ radiation enters a collimator (Baffle) through an optical interference filter with a bandwidth of about $10 \mathrm{~nm}$ (FWHM) centered at about $120 \mathrm{~nm}$. The collimator is made of blackened aluminum honeycomb material, with a length of $2.54 \mathrm{~cm}$ and a cell pitch of $1.53 \mathrm{~mm}$, defining an almost circular field-of-view (FOV) of approximately $4^{\circ}$. The Lyman- $\alpha$ radiation is then detected by a channeltron multiplier with an attached amplifier/discriminator circuit. The output pulse frequency of the discriminator is proportional to the Lyman- $\alpha$ intensity and is determined by a digital pulse 


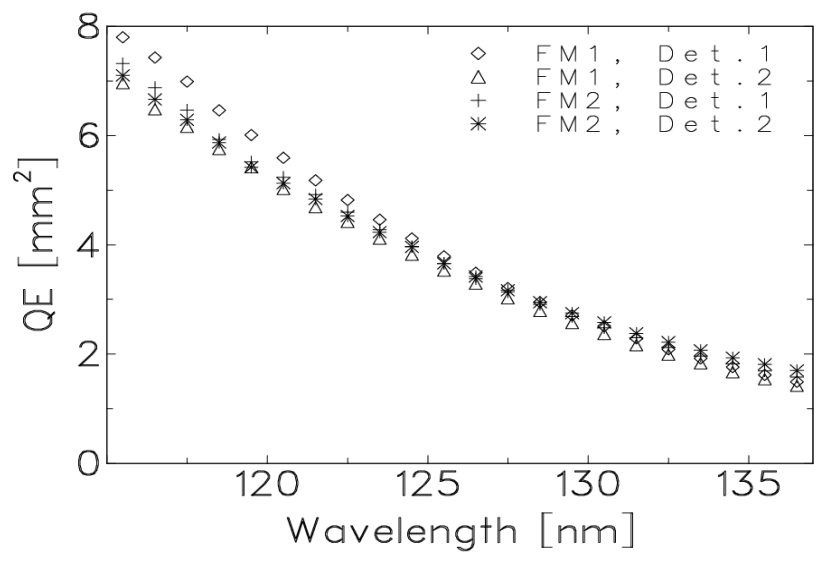

Fig. 5. Total quantum efficiency QE for all 4 LAD sensors (without filter).

counter. The company von Hörner and Sulger (vH\&S) in Schwetzingen, Germany was the main contractor, and did all development, manufacturing, and qualification of the LAD instruments, while the EBOX as part of the TWINS instrumentation is supplied by the payload.

\section{Calibration}

All four Lyman- $\alpha$ sensors were calibrated at the Berlin Electron Synchrotron (BESSY II) in Berlin-Adlershof, Germany. The synchrotron was operated at very low ring currents some $\mu \mathrm{A}$ - yielding a Lyman- $\alpha$ radiation at an intensity range of the expected geocoronal values. In Fig. 4 the result for one of the four detectors is given. Shown is the local quantum efficiency $\mathrm{QE}^{\prime}$ at Lyman- $\alpha$ measured across the entrance area of the channeltron given in cts per 100 photons, i.e. percent. As can clearly be seen, the efficiency is best near the center, i.e. close to the anode and decreases towards the edge of the channeltron. The total quantum efficiency $Q E(\lambda)$ of the whole surface is given by

$Q E(\lambda)=\int Q E^{\prime}(\lambda) d A$,

where the integration has to be performed over the whole detector surface (circular, diameter $2 \mathrm{~cm}$ ). These total quantum efficiencies of all four detectors (without interference filters) are plotted as a function of the wavelength in Fig. 5. It is evident that all four channeltrons produce roughly the same output. This changes a little bit - shown in Fig. 6 when the complete sensor, i.e. channeltron plus filter is considered, caused by slight differences in the filter transmissivities. The peak of the detector sensitivities is shifted to $115 \mathrm{~nm}$, say, due to the folding of the filter transmissivity $\operatorname{Tr}(\lambda)$ (almost a Gaussian centered at Lyman- $\alpha$ ) with the total quantum efficiency $Q E(\lambda)$ shown in Fig. 5. Looking through the exosphere in the above described geometry

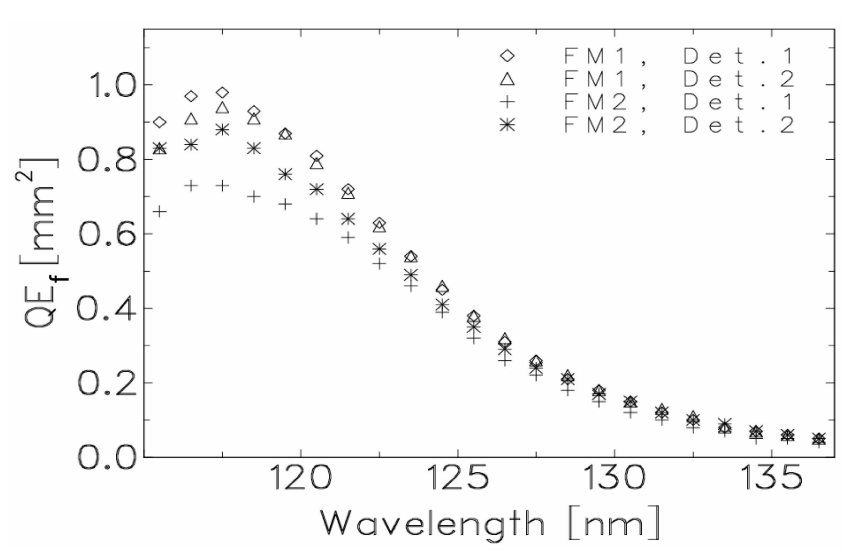

Fig. 6. Total quantum efficiency $Q E_{f}$ for all $4 \mathrm{LAD}$ sensors (with filter).

almost only backscattered solar Lyman- $\alpha$ radiation will be measured by the detectors. A potential contribution of the oxygen $130.4 \mathrm{~nm}$ radiation will effectively be suppressed by a factor of $4-5$, as can be seen in Fig. 6, though for the height regime which is primarily of interest $(\geq 2.9$ earth radii, see Sect. 1) this contribution will be negligible. Usually airglow emission rates are given in units of rayleighs $(\mathrm{R})$, where $1 R=10^{6} /(4 \pi) \mathrm{phot} /\left(\mathrm{cm}^{2} \mathrm{~s}\right.$ steradian $)$. For the LAD detectors with an aperture angle of $4^{\circ}$ one thus gets

$$
1 R \triangleq \frac{10^{6}}{4 \pi} \times 2 \pi\left[1-\cos \left(\frac{4^{\circ}}{2}\right)\right] \times Q E_{f}(121.6) \frac{\mathrm{cts}}{\mathrm{s}}
$$

which yields a conversion factor of approximately $2 \mathrm{cts} / \mathrm{s} / \mathrm{R}$ for all 4 detectors. Since the geocoronal Lyman- $\alpha$ intensity is to be expected in the range of some thousand Rayleighs resulting in a detector signal of some 2 thousand cts/s, and in view of the measured detector noise level of less than $1 \mathrm{cts} / \mathrm{s}$, a signal to noise ratio of $>2 \times 10^{3}$ is accomplished.

\section{Geocoronal Model}

In order to deconvolute the three dimensional geocoronal hydrogen distribution from Lyman- $\alpha$ line-of-sight (LOS) measurements a theoretical/empirical hydrogen model is needed. One mathematical advanced model that is suitable for this purpose was published by Hodges (1994). It not only tries to model mathematically the radial, but also the longitudinal and latitudinal density distribution of the hydrogen geocorona. Furthermore, its mathematical formulation can easily be adapted to what may be suggested by the measurements.

In detail, this model describes the hydrogen exosphere up to $10 R_{e}$ based on a Monte-Carlo simulation and includes a comprehensive number of relevant scattering-, gain- and 


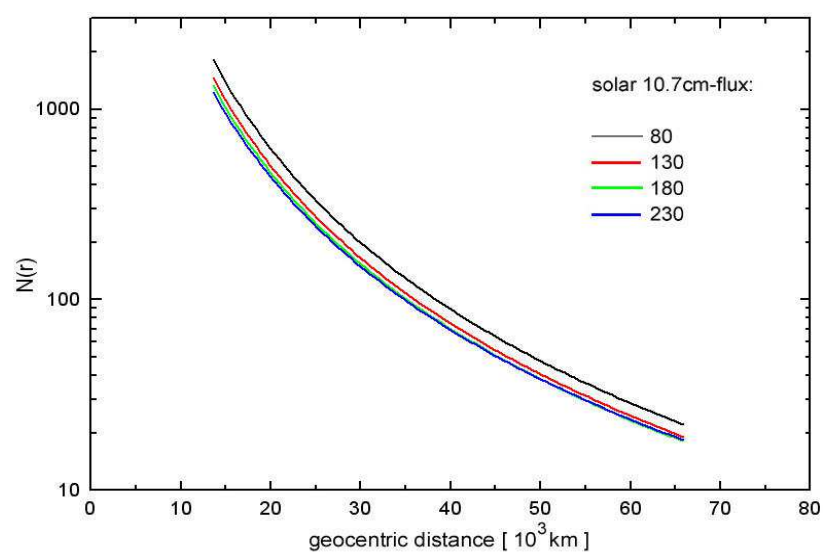

Fig. 7. Hodges' parameter $N(r)$ for equinox conditions.

loss effects of different coupling regions. Thermal and nonthermal scattering- and charge-exchange processes are considered. An exosphere/plasmasphere coupling is included as well as solar wind interactions in the magnetospheric cavity and at its boundary.

The model hydrogen densities are in good agreement with IMAGE-observations below $6 R_{e}$ (Østgaard et al., 2003). Hdensity ratios between the equatorial night- and day side or the pole/equator-asymmetry calculated with the Hodges model are also in good agreement with observations from Brinton and Mayr (1971) and Vidal-Madjar et al. (1973).

The Hodges model comes formally as a third order spheric harmonic expansion with the altitude-dependent coefficients $N(r), A_{l m}(r)$ and $B_{l m}(r)$ :

$n_{H}(r, \phi, \theta)=N(r) \sqrt{4 \pi} \sum_{l=0}^{3} \sum_{m=0}^{l} Z(r, \phi, \theta)$,

with

$Z(r, \phi, \theta)=\left[A_{l m}(r) \cos (m \phi)+B_{l m}(r) \sin (m \phi)\right] Y_{l m}(\theta)$,

where $Y_{l m}(\theta)$ are the spherical harmonic Legendre functions.

Hodges published results for the above mentioned coefficients $N(r), A_{l m}(r)$, and $B_{l m}(r)$ for 2 different earth positions (solstice and equinox) using 4 different solar $10.7 \mathrm{~cm}$ fluxes $\left(80,130,180,230\left[\times 10^{-22} \mathrm{Ws} / \mathrm{m}^{2}\right]\right)$. The geocentric distance $r$ is separated in 40 nonequidistant steps. This results in a total of $16 \times 40=640$ coefficients for each solar flux value and orbital position of the earth. (At the equinox position the sun is above the earth equator, at solstice above the tropic of cancer or the tropic of capricorn, respectively.)

An upcoming problem with the usage of the mentioned Hodges model for TWINS purposes was to handle the huge amount of free coefficients within the procedure to fit the hydrogen density distribution ( $16 \times 9=144$, if only heights

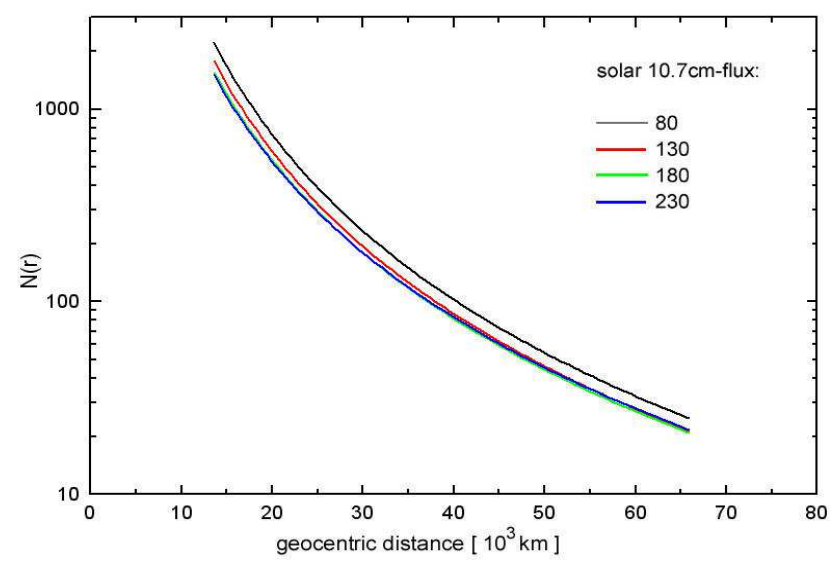

Fig. 8. Hodges' parameter $N(r)$ for solstice conditions.

relevant for the TWINS mission are considered for a specific earth position and $10.7 \mathrm{~cm}$-flux).

A detailed inspection of the $r$-dependence of the Hodgescoefficients $N(r), A_{l m}(r)$ and $B_{l m}(r)$ allowed for the following simplifications to the model without a significant deviation in the resultant density distribution for the heights relevant to the TWINS mission $(r \geq 15000 \mathrm{~km})$ :

- For all fluxes and earth positions discussed by Hodges the coefficient $N(r)$ shows the same functional relationship according to

$N(r)=a \times r^{b}$,

which is shown in Figs. 7 and 8 for equinox and solstice conditions, respectively.

- The coefficients $A_{l m}(r)$ can be set to

$$
A_{l m}(r)=a_{l m}+b_{l m} \times r .
$$

- The coefficients $B_{l m}(r)$ can be set to

$$
B_{l m}(r)=0 .
$$

- The order of the expansion can be reduced from 3 to 2 .

The newly introduced coefficients $\left(a, b, a_{l m}\right.$ and $\mathrm{b}_{l m}$ for $l=1,2$ and $m \leq l)$ are, of course, different for each solar flux and orbital position of the earth.

By these measures the number of coefficients to be fitted was reduced from 144 to only 12 yielding a very stable behavior of the fitting procedure.

In order to test the usability and the quality of the fitting procedure Lyman- $\alpha$ LOS intensities were calculated on the basis of the Hodges model with feasible geocoronal and solar parameters assuming a suitable Molnya-type orbit. To 


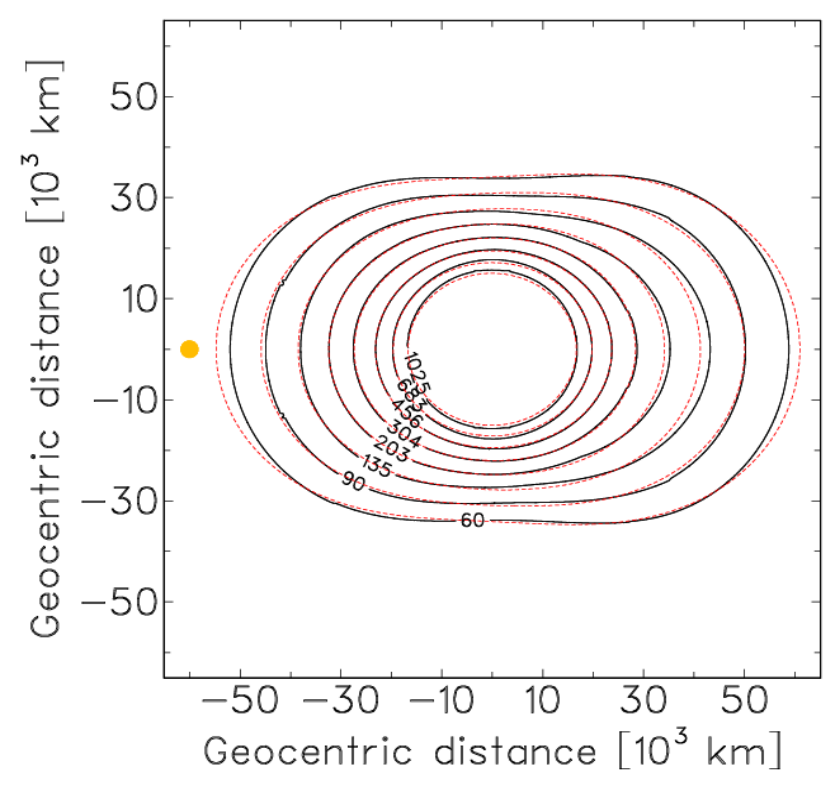

Fig. 9 Shown is for equinox conditions and $f_{10.7}=130$ in the midnight meridian plane the geocoronal hydrogen density distribution (red lines) as obtained by the described fitting procedure. This compares quite well to the original Hodges densities (black lines). The direction to the sun is indicated by the yellow dot (not to scale!).

these intensities some interplanetary background (based on a standard hot model) was added. These total intensities then were converted into count rates according to the instrumental calibration factors. Addition of some instrumental random noise yielded a simulated LAD data set. This data set then was used as input for a least square fit procedure in order to determine the above mentioned coefficients $\left(a, b, a_{l m}\right.$ and $\left.b_{l m}\right)$. Figures 9 and 10 show the comparison between the original (Hodges model) and the fitted regenerated density distribution, in the midnight meridian plane for equinox and solstice conditions, respectively. For all cases considered by Hodges the agreement between his model and the here discussed modified model is sufficiently good to allow the modelling of the geocoronal hydrogen density on the basis of the TWINS-LAD data. It should be mentioned that only the data of half an orbit are sufficient to generate a complete density distribution.

\section{Conclusions}

It was shown that fitting the Lyman- $\alpha$ measurements of the TWINS-LAD experiment yields an exospheric hydrogen distribution for each specific day, based on a modified Hodges model. The model coefficients, of course, will depend on solar flux and orbital position of the earth, i.e. vary with time. These dependencies will be determinable during the TWINS

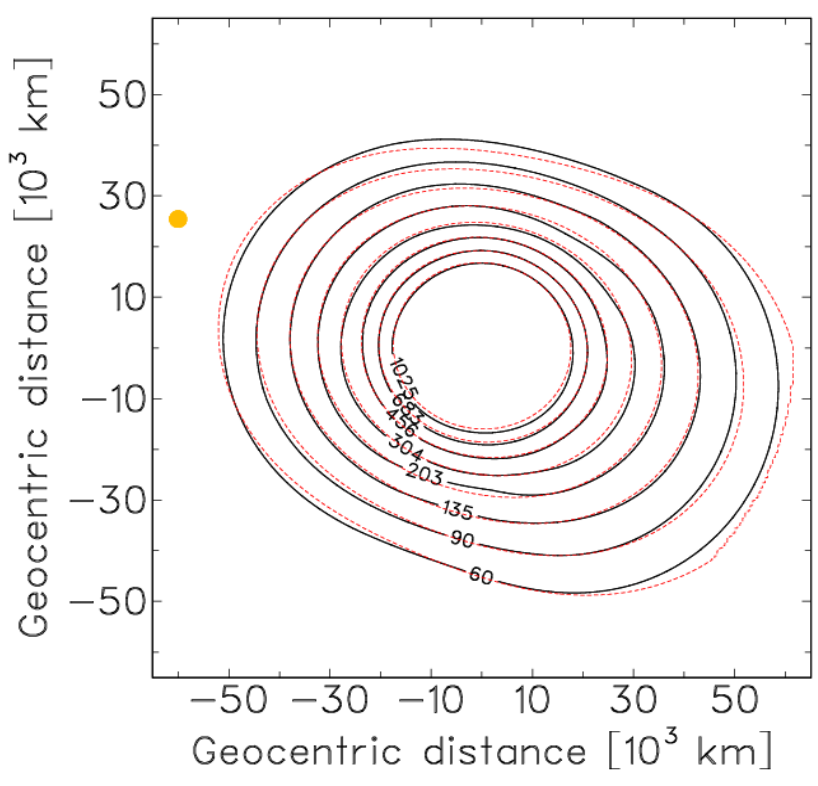

Fig. 10 Same as Fig. 9, but for solstice conditions. The direction to the sun is indicated by the yellow dot (not to scale!).

mission which is to last for at least 3 years. It is hoped that the development of a new improved empirical model of the hydrogen geocorona will be possible on the basis of the measurements during this mission.

Acknowledgements. This work was supported by the German DLR (project no. 50 OE0001). We are grateful to the referees for helpful remarks and comments.

Edited by: M. Gruntman

Reviewed by: R. R. Hodges, Jr. and N. Østgaard

\section{References}

Brinton, H. C. and Mayr, H. G.: Temporal variations of thermospheric hydrogen derived from in situ measurements, J. Geophys. Res., 76, 6198-6201, 1971

Hodges, Jr., R. R.: Monte Carlo simulation of the terrestrial hydrogen exospere, J. Geophys. Res., 99, 23 229-23 247, 1994

Østgaard, N., Mende, S. B., Frey, H. U., Gladstone, G. R., and Lauche, H.: Neutral hydrogen density profiles derived from geocoronal imaging, J. Geophys. Res., 108AJ, SMP 18-1, doi:10.1029/2992JA009749, 2003

Vidal-Madjar, A., Blamont, J. E., and Phissamay, B.: Solar LyAlpha changes and related hydrogen density distribution of the earth's exobase (1969-1970), J. Geophys. Res., 78, 1115-1144, 1973 